\title{
DESIGN OF CANDIDA ANTARCTICA LIPASE B THERMOSTABILITY IMPROVEMENT BY INTRODUCING EXTRA DISULFIDE BOND INTO THE ENZYME
}

\author{
Usman Sumo Friend Tambunan, Ahmad Randy and Arli Aditya Parikesit \\ Department of Chemistry, Faculty of Mathematics and Science, University of Indonesia, Depok 16424, Indonesia
}

Received 2014-03-20; Revised 2014-04-13; Accepted 2014-04-18

\begin{abstract}
Candida Antarctica Lipase B (CALB) is extensively studied in enzymatic production of biodiesel, pharmaceutical products, detergents and other chemicals. One drawback of using CALB is its relatively low optimum temperature at $313 \mathrm{~K}\left(40^{\circ} \mathrm{C}\right)$. The objective of this research is to design CALB mutant with improved thermostability by introducing extra disulfide bond. Molecular dynamic simulation was conducted to get better insight into the process of thermal denaturation or unfolding in CALB. Thermal denaturation of CALB was accelerated by conducting simulation at high temperature. Molecular dynamic simulation of CALB was performed with GROMACS software package at 300-700 K. Prediction of possible mutation was done using "Disulfide by Design ${ }^{\mathrm{TM}}$ ", software. Selection of mutated residues was based on flexibility analysis of CALB. From those analyses, three mutants were designed, which are Mutant-1 (73LeuCys/151AlaCys), Mutant-2 (155TrpCys/294GluCys) and Mutant-3 (43ThrCys/67SerCys). Parameters that were used to compare the thermostability of mutant with wild type enzyme were Root Mean Square Deviations (RMSD), Solvent Accessible Surface Area (SASA), Radius of gyration (Rg) and secondary structure. Molecular dynamic simulation conducted on those three mutants showed that Mutant-1 has better thermostability compared to wild type CALB. We proposed the order of mutant thermostability improvement as follows: Mutant-1, Mutant-2 and Mutant-3, with Mutant-1 having better potential thermostability improvement and Mutant-3, the least stable.
\end{abstract}

Keywords:Candida antarctica Lipase B, Thermostability, Molecular Dynamic Simulation, Mutation, Disulfide Bond

\section{INTRODUCTION}

Lipase is a group of enzyme, which members are utilized in many fields of application such as production process of detergent, surfactant, food, pharmaceutical product, cosmetic, paper and pulp and nutrition (Sharma et al., 2001; Bisht et al., 2012). According to Ganjalikhany et al. (2012), Lipase B of Candida antarctica (C. antarctica lipase B, CALB) is extensively studied in catalyzing chemical reaction such as resolution of racemic compounds (Patel et al., 2000), biodiesel production (Zhao et al., 2007) and ringCorresponding Author: Usman Sumo Friend Tambunan, Department
University of Indonesia, Depok 16424, Indonesia

opening polymerization (Peeters et al., 2005). CALB is highly enantioselective, can utilize wide range of substrate and has a good stability in organic medium (Ong et al., 2006). However, the drawback of using CALB is its low thermostability, where the optimum working temperature of $\mathrm{CALB}$ is at $40^{\circ} \mathrm{C}$ and its stability and activity decrease at higher temperature (Mittelbach, 1990; Xu et al., 2003; Suen et al., 2004; Chodorge et al., 2005; Ong et al., 2006). Enzyme with high thermostability is more favored because many industrial processes require higher temperature to increase the reaction rate, decrease substrate solubility University of Indonesia, Depok 16424, Indonesia 
and reduce contamination (Haki and Rakshit, 2003; Ong et al., 2006).

Mutation of protein via genetic engineering to change its amino acid sequences is one strategy that can be utilized to improve certain protein properties such as thermostability (Subramanian and Natarajan, 2011). One mutational strategy to engineer a protein in order to improve its thermostability is by introducing new/extra disulfide bond into the protein molecule (Ling et al., 2009). This is conducted by mutating two (a pair) amino acid residues to cysteine (Tambunan et al., 2011b). Successful examples of thermostability improvement by adding new disulfide bond were phage T4 lysozyme (Matsumura et al., 1989) and Bacillus circulans xylanase (Wakarchuk et al., 1994). Disulfide bond is known to stabilize protein both entalphically and entropically. Decreasing denatured state entropy can be considered as the major effect of introducing disulfide bond, thus favouring the native state (Betz, 1993; Yamaguchi et al., 1996; Babakhouya et al., 2010), although enthalpic stabilization of native state should also be considered (Betz, 1993). Analyzing flexible area in protein that shows high movement can be utilized to determine potential mutation target. This highly flexible area is considered to play a role in early unfolding process and improving rigidity of this area, e.g., introducing disulfide bond can potentially quench unfolding process (Liu and Wang, 2003).

Recent developments in computational biology such as molecular dynamic simulation and molecular modeling can help the task on engineering certain protein properties (Vijayan et al., 2007). Computer aided design has become a common practice now in protein engineering, especially as an initial step to design a mutant enzyme and to simulate certain properties of the mutant (Yamaguchi et al., 1996; Santarossa et al., 2005; Sandström et al., 2009; Han et al., 2009; Kim et al., 2010). Molecular dynamic simulation helps researcher to study the physical changes in protein in their response to temperature changes (Li and Daggett, 1994; Day et al., 2002; Liu and Wang, 2003; Purmonen et al., 2007; Paschek et al., 2011). Analysis of flexible area in protein during temperature changes can be performed by molecular dynamic simulation.

The objective of this study is to design CALB mutant that has improved thermostability by introducing extra disulfide bond. Molecular dynamic simulation was utilized to analyze protein structural changes during elevated temperature and analyze the thermostability of mutants represented by several physical properties (Harishchander et al., 2010). Thermal denaturation of
CALB was accelerated by conducting simulation at high temperature. Obtained mutant design could be proposed to be implemented for site-directed mutagenesis of CALB.

\section{MATERIALS AND METHODS}

\subsection{Molecular Dynamic Simulation}

Molecular dynamic simulation was conducted with GROMACS package (Van Der Spoel et al., 2005; Hess et al., 2008) running in Ubuntu 10.04 LTS. Model visualization and analysis was conducted with GROMACS package and VMD 1.9 (Humphrey et al., 1996). CALB crystal structure was PDB 1TCA (Uppenberg et al., 1994) with every water molecule and $\mathrm{N}$-acetyl-D-glucosamine in the crystal removed prior to simulation. AMBER99 (Wang et al., 2000) force field was used to define potential energy of the model. Solvation of the system was conducted using TIP3P water molecule (Jorgensen et al., 1983). Wild type CALB simulation system was prepared in a dodecahedron box with total volume of $350.77 \mathrm{~nm} 3$. Sodium ion was added to neutralize the total charge. This whole system consisted of 1 CALB molecule, 10284 TIP3P water molecule and one sodium ion.

\subsection{System Minimization}

System minimization was conducted in two steps: 1000 steps of steepest descent, followed by 1000 steps of conjugate gradient (Payne et al., 2013). Minimization was followed by short simulation for 10 ps with positional restraint on protein molecules. NVT ensemble was used in simulation by coupling the system to velocity rescaling thermostat using coupling constant of 0.1 (Bussi et al., 2007; Larsson et al., 2012). Electrostatic and van der Waals interactions were modeled with Particle Mesh Ewald (PME) (Essman et al., 1995) and Lennard-Jones potential, respectively. Each was calculated in $1.0 \mathrm{~nm}$ cut-off. LINCS algorithm (Hess et al., 1997) was used to constrain every bond that involves hydrogen atom.

Main simulation steps were heating to desired temperature for $10 \mathrm{ps}$, followed by $100 \mathrm{ps}$ equilibration and production for 6000 ps. Average computational speed was around $2.3 \mathrm{~ns} /$ day; thus 6000 ps simulation time would take about 3 days of real computing time.

\subsection{Mutation of the Amino Acids}

Candidate of amino acid residues that can be mutated to cysteine were created by Disulfide by Design ${ }^{\mathrm{TM}}$ software (Benson and Daggett, 2008). The crystal 
structure of CALB after minimization step was used as an input for this software. Parameters for creating mutation candidate were Optimum Chi3-angle = $+100^{\circ} /-80^{\circ}$, tolerance $\pm 10^{\circ}$; and $\mathrm{Ca}$ - $\mathrm{Cb}$-S-angle $=$ $114.60^{\circ}$, tolerance $\pm 10^{\circ}$. Model of CALB mutant molecules was also generated using this software by selecting desired pair of amino acids. Mutant models were prepared in same simulation system as the wild type CALB. Sodium or chloride ion was added when necessary as counter ion to neutralize the total charge of simulation system (Senn and Thiel, 2009). Simulation of mutants CALB was also done using the same minimization steps and simulation parameters.

\section{RESULTS}

\subsection{Validation of Model}

Validation of a simulation system or model can be done by comparing simulation data with experimental data (Chandramat et al., 2005; Lim et al., 2010). Some simulation data that can be used are density, salvation free energy, viscosity, surface tension and crystallographic Bfactor (Pikkemaat et al., 2002). In this study, B-factor from experimental data of PDB 1TCA was used and compared with B-factor from simulation. We used C- $\alpha$ atoms RMSF from simulation of wild type CALB at 300 $\mathrm{K}$, specifically from $4000-6000 \mathrm{ps}$. Bfactors values were determined from RMSF value using the following equation (Kuzmanic and Zagrovic, 2010):

$$
\operatorname{RMSF}_{\mathrm{i}}^{2}=\frac{3 \mathrm{Bi}}{8 \pi^{2}}
$$

Figure 1 shows crystallographic B-factor and simulation B-factor. Overall observation shows that most crystallographic and simulation B-factor values do not differ significantly and both shows similar pattern. Large difference was observed for residues 4-9, 12-20, 141-150 and 285-289. This particularly resulted from the different conditions of B-factor obtained. Simulation was performed on a solvated model, where protein molecules got more flexible and more protein movement was expected compared to more rigid crystal state. Overall, we conclude that this model has good validity to be able to represent desired experiment.

\subsection{Mutant Selection}

Mutant selection was based on flexibility analysis by considering RMSF analysis and mutant prediction by Disulfide by Design ${ }^{\mathrm{TM}}$ software (Subramanian and
Natarajan, 2011). First, we determined highly flexible residue in CALB as mutation candidate. Highly flexible residues were generally considered to play a role in early unfolding process. Introducing more rigid property, like building a disulfide bond, would limit the movement in this flexible area and help in quenching the transition to unfolded state (Pikkemaat et al., 2002; Liu and Wang, 2003; Jeong et al., 2007; Han et al., 2009). Here, we use RMSF value to represent atomic movement in the backbone atoms, especially $\mathrm{C}-\alpha$ atoms in varied simulation temperatures.

Simulation on varied temperature shows that higher temperature gave larger RMSF value (Fig. 2). We conducted simulations up to $700 \mathrm{~K}$, but here we only used RMSF value up to $400 \mathrm{~K}$. We set a minimal RMSF value of $0.05 \mathrm{~nm}$ as a criterion where CALB is still considered in the native state (Benson and Daggett, 2008). We exclude the RMSF data of $450-700 \mathrm{~K}$ simulation because we consider that CALB molecules in these simulations were already in the unfolded state (all RMSF values $>0.5$ ) and include RMSF data of 300-400 $\mathrm{K}$ because they still have some residues with RMSF value of $\leq 0$. There are nine residue areas that have high flexibility: Residues 1-30, 43-62, 91-97, 140-153, 168173, 184-203, 210-225, 238-297 and 304-317.

RMSF value was taken from simulation at 4000-6000 ns of each temperature. Disulfide by Design ${ }^{\mathrm{TM}}$ was used to generate pair of residues that are likely to form disulfide bond when mutated to cysteine based on geometric properties of disulfide bond such as $\mathrm{C} \beta-\mathrm{S} \gamma$ and $\mathrm{S} \gamma-\mathrm{S} \gamma$ bond length and $\mathrm{C} \beta-\mathrm{S} \gamma-\mathrm{S} \gamma$ angle (Dombkowski, 2003). The software predicted twelve pairs of residues in CALB that could be mutated to cysteine and form disulfide bond (Table 1). On the other hand, it can also analyze three disulfide bonds that already exist in CALB, which prove good validity of the software. There were no residues suggested that involved in catalytic, binding or oxyanion hole sites. Results from flexibility analysis and software prediction were combined to generate CALB mutants. We exclude residues that are assigned in the middle of helix or strand structure because adding disulfide bond to these structure would add extra restrain on the structure (Pikkemaat et al., 2002). Matching these three criteria (flexibility, not assigned in secondary structure and software output), we obtained three pairs of potential mutation to cysteine, which are: 73Leu-151Ala, 155Trp-294Glu and 43Thr-67Ser. Three mutant models were generated using Disulfide by Design ${ }^{\mathrm{TM}}$ from those mutations: Mutant-1 (Leu73Cys/Ala151Cys), Mutant-2 (Trp155Cys/Glu294Cys) and Mutant-3 (Thr43Cys/Ser67Cys). 


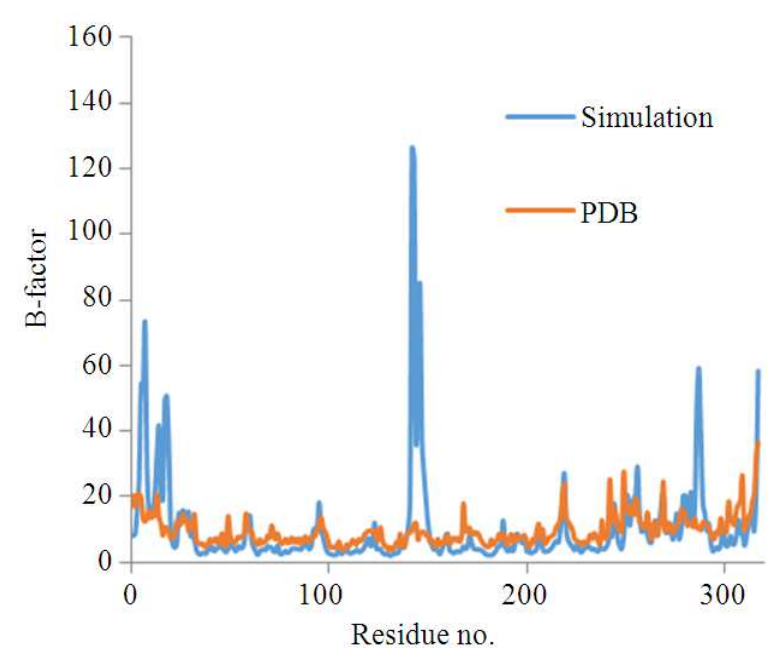

Fig.1. Crystallographic B-factor experiment (PDB 1TCA) compared to simulation B-factor

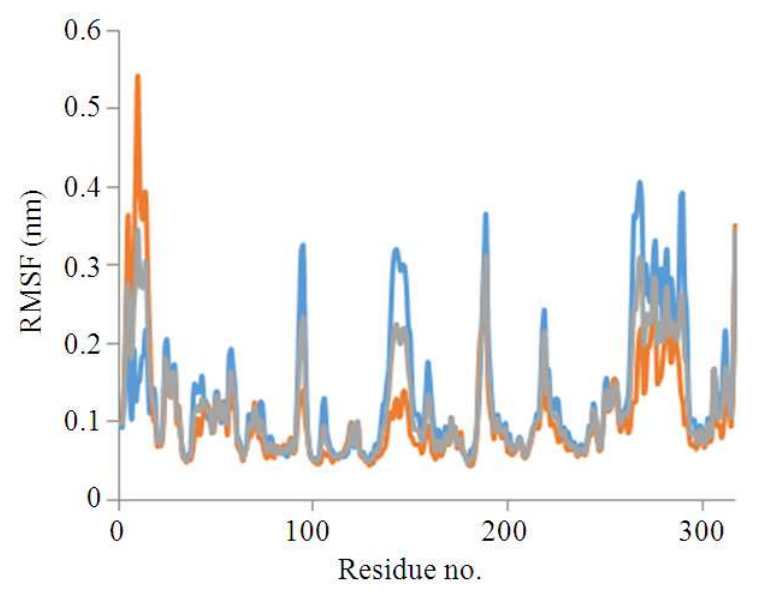

Fig. 2. RMSF analysis of CALB simulation $300 \mathrm{~K}$ (orange), $350 \mathrm{~K}$ (grey), and $400 \mathrm{~K}$ (blue)

Table 1. Software prediction (disulfide by Design ${ }^{\mathrm{TM}}$ ) of pair of residues that can likely form disulfide bond when mutated to cysteine

\begin{tabular}{l}
\hline Parameter \\
\hline 42Thr-46Gln \\
43Thr-67Ser \\
50Ser-274Ala \\
73Leu-151Ala \\
155Trp-94Glu \\
167Leu-72Gly \\
183Tyr-202Ser \\
208Lys-47Gln \\
234Tyr-63Ala \\
239Ser-52Asp \\
266Leu-70Gln
\end{tabular}

\subsection{Molecular Dynamics Simulation of Mutants}

All three mutants were simulated at 450 and $550 \mathrm{~K}$ simulation temperature. These two temperatures were chosen because at $450 \mathrm{~K}$, the physical parameters (radius of gyration and solvent accessible surface area) of wild type CALB just started to give significant increase, while at $550 \mathrm{~K}$ rapid structural changes were observed (Fig. 3) (Ceruso et al., 2004). Thus, $450 \mathrm{~K}$ simulation represents the start of unfolding process and $550 \mathrm{~K}$ simulation represents the unfolded state. We compare the physical parameter (RMSD, SASA and Rg) of wild type and mutants to represent the thermostability of both molecules (Gundampati et al., 2013). Significant increase in Solvent Accessible Surface Area (SASA) will happen when a protein molecule starts to lose its stabilizing interactions (intermolecular hydrogen bond, electrostatic and van der Waals interaction) and hydrophobic core of protein molecule starts to collapse; thus the protein will be more readily accessible to the solvent molecule (Day et al., 2002; Yan et al., 2010). According to this explanation, more linear SASA value shows more stability in the protein structure along simulation time. Radius of gyration ( $\mathrm{Rg}$ ) can also be used to relate protein stability in terms of unfolding process (Li and Daggett, 1994; Day et al., 2002; Zhao et al., 2009; Paschek et al., 2011).

RMSD analysis on Mutant-1 and wild type on 450 and $550 \mathrm{~K}$ simulation showed that Mutant-1 can maintain its structural stability better compared to wild type (Fig. 4). In both temperatures, final RMSD value of Mutant-1 is smaller than wild type. At $450 \mathrm{~K}$ simulation, Mutant-1 reaches linearity faster than wild type. This indicates that Mutant-1 is capable of maintaining its structural stability better than wild type because RMSD represents the structural differences in each simulation time compared to its initial structure. Investigation on SASA and $\mathrm{Rg}$ value also indicates the stability of Mutant-1 relative to wild type (Fig. 5 and 6). There was no significant difference at $450 \mathrm{~K}$ simulation; however, Mutant-1 maintains its structural stability better at $550 \mathrm{~K}$. This is shown by the ability of Mutant-1 to maintain the linear trend of RMSD longer. At $550 \mathrm{~K}$, wild type CALB started to give a jump in RMSD value at $1000 \mathrm{ps,}$, while Mutant-1 still gave linear RMSD up to 4000 ps.

Mutant-2 also shows relative stability to wild type for RMSD value at $450 \mathrm{~K}$ simulation; meanwhile at $550 \mathrm{~K}$ the RMSD of both did not differ significantly (Fig. 7). 

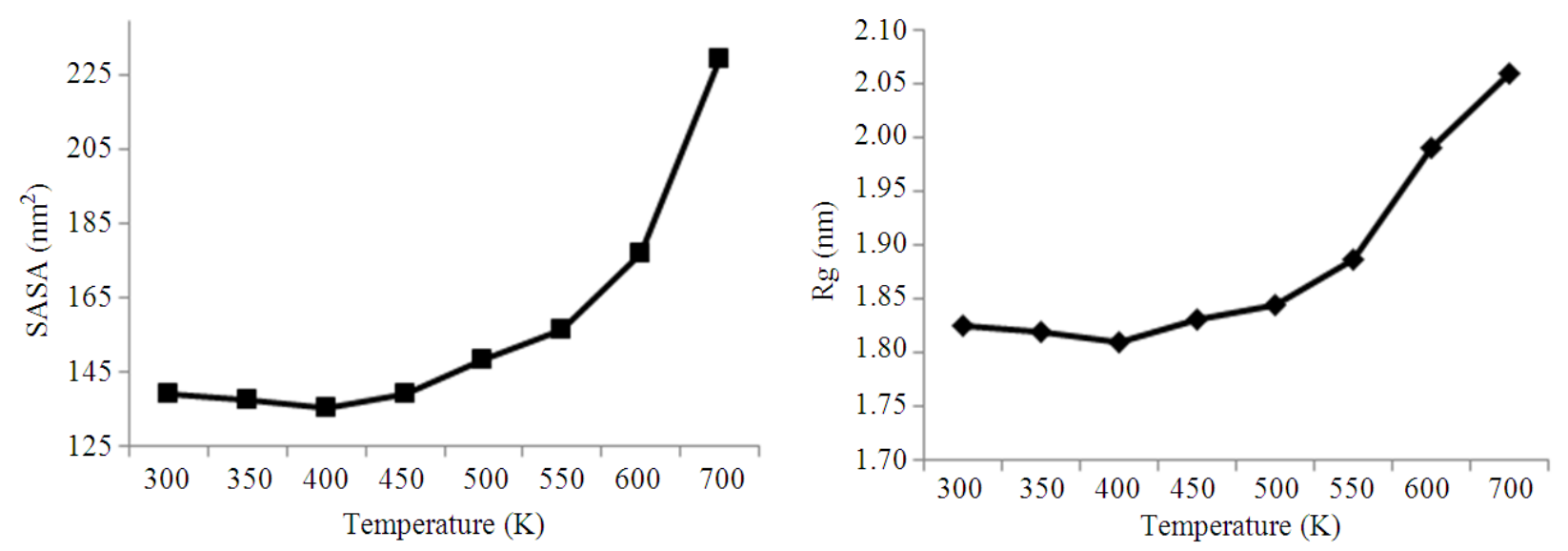

Fig.3. Average solvent accessible surface area. (Up) Average Solvent Accessible Surface Area (SASA) of final 2000 ps simulation of wild type CALB; and (Down) average Radius of gyration (Rg) of final 2000 ps simulation of wild type CALB

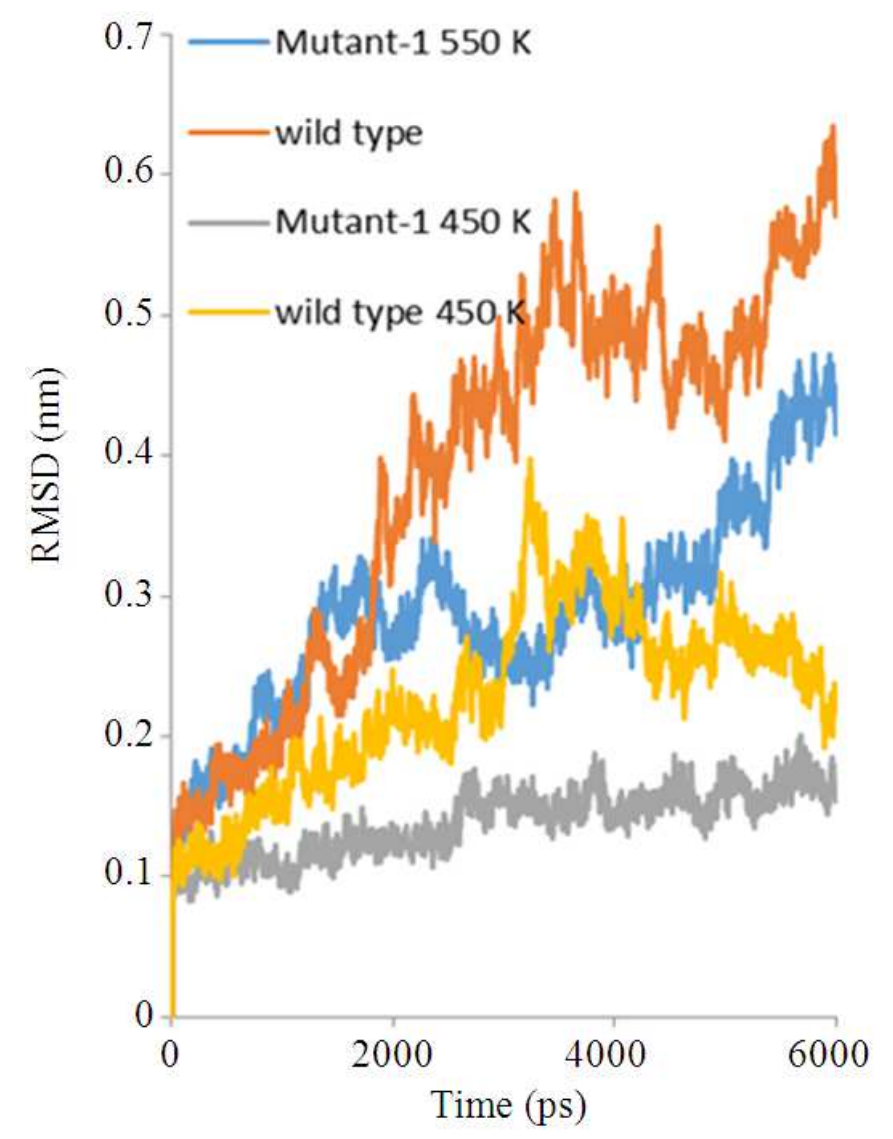

Fig. 4. RMSD value of Mutant- 1 and wild type CALB at 450 and $550 \mathrm{~K}$ simulation.Mutant-1 at $550 \mathrm{~K}$ (blue), wild type CALB at $550 \mathrm{~K}$ (orange), Mutant-1 at $450 \mathrm{~K}$ (grey), wild type CALB at $450 \mathrm{~K}$ (yellow) 

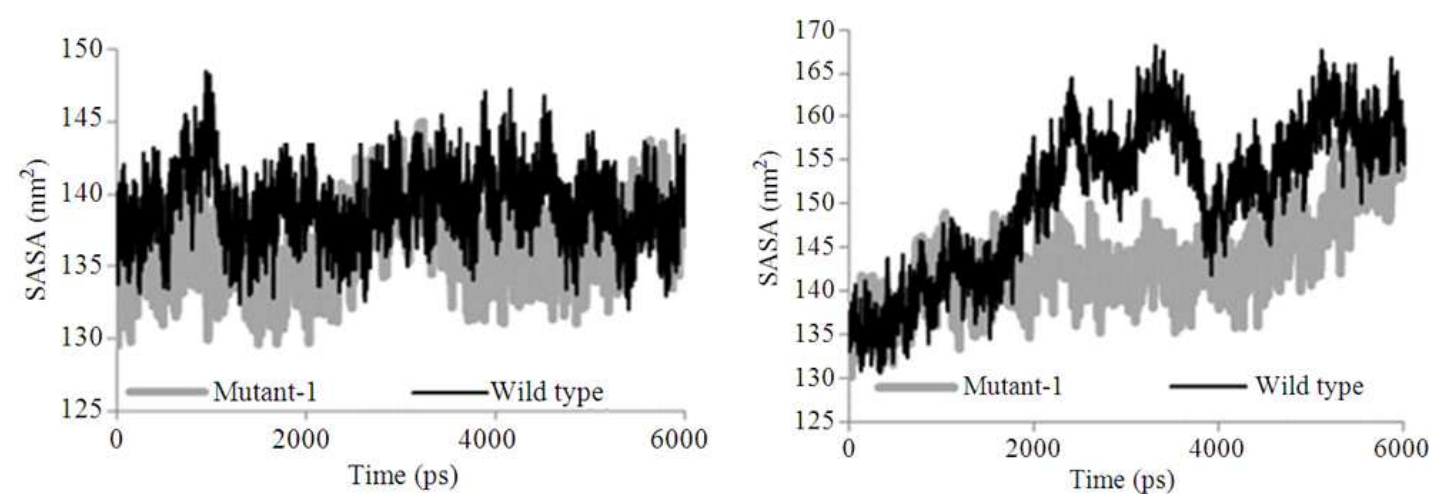

Fig. 5. SASA value of Mutant-1 and wild type CALB $450 \mathrm{~K}$ (up) and $550 \mathrm{~K}$ (down) simulation
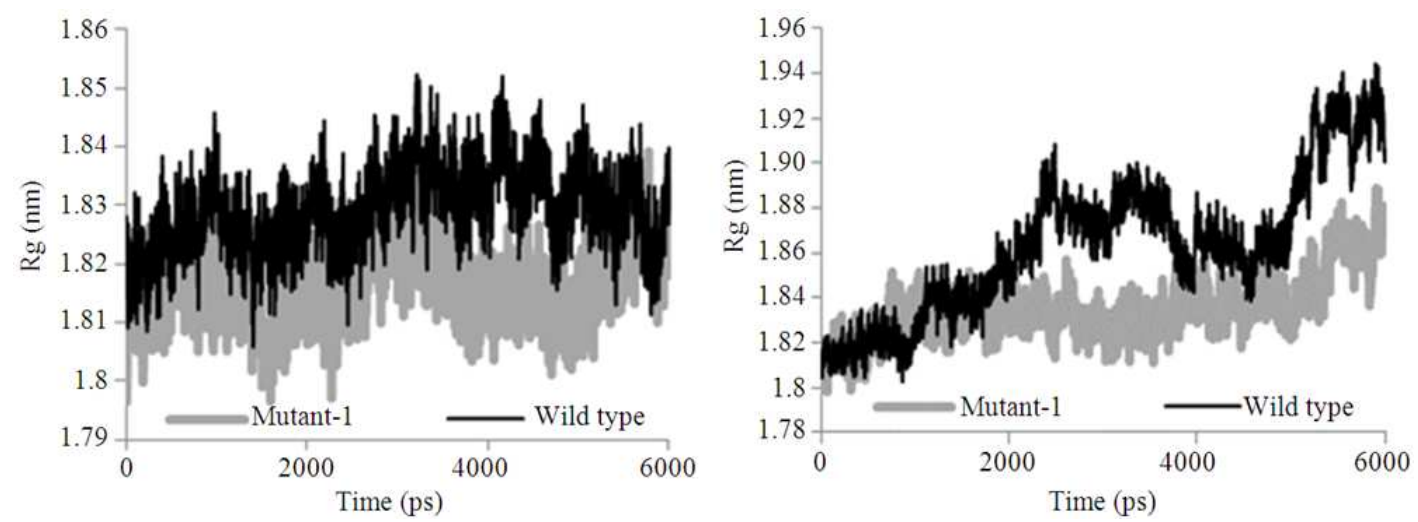

Fig. 6. $\mathrm{Rg}$ value of Mutant-1 and wild type CALB $450 \mathrm{~K}$ (up) and $550 \mathrm{~K}$ (down) simulation

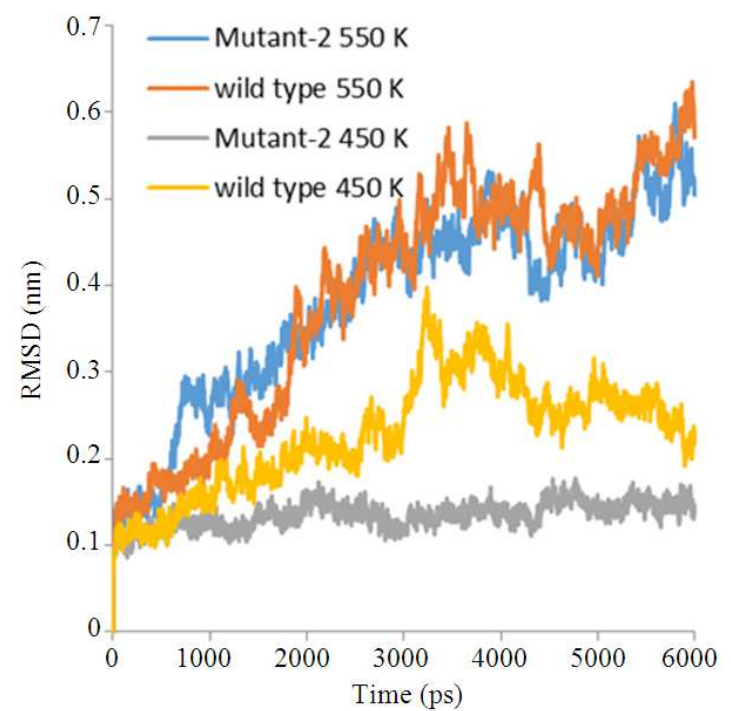

Fig. 7. RMSD value of Mutant-2 and wild type CALB at 450 and $550 \mathrm{~K}$ simulation Mutant-2 at $550 \mathrm{~K}$ (blue), wild type CALB at $550 \mathrm{~K}$ (orange), Mutant-2 at $450 \mathrm{~K}$ (grey), wild type CALB at $450 \mathrm{~K}$ (yellow)
This might suggest that Mutant-2 was more thermostable than wild type, but not as stable as Mutant-1. Further analysis on SASA and Rg also supports this conclusion. At $450 \mathrm{~K}$, both SASA and Rg values of Mutant- 2 and wild type show close value between them (Fig. 8 and 9). At 550, the final SASA and Rg values of Mutant-2 and wild type were close to each other; although along the simulations there were some period of times that Mutant2 gave lower value of SASA and $\mathrm{Rg}$. All of these analyses might support the conclusion that Mutant-2 still has better thermostability than wild type, but less stable compared to Mutant-1.

Among the three generated mutants, Mutant-3 shows the least stability. Simulation of Mutant-3 at $450 \mathrm{~K}$ shows that Mutant-3 indeed maintains its stability by slowly increasing RMSD value, although final RMSD values were close to each other (Fig. 10). At $550 \mathrm{~K}$, both RMSD along simulation did not differ much; in fact, the final RMSD value of Mutant-3 exceeded the wild type. Meanwhile, the SASA and Rg value of Mutant-3 did not differ from the wild type at both simulation temperatures. 

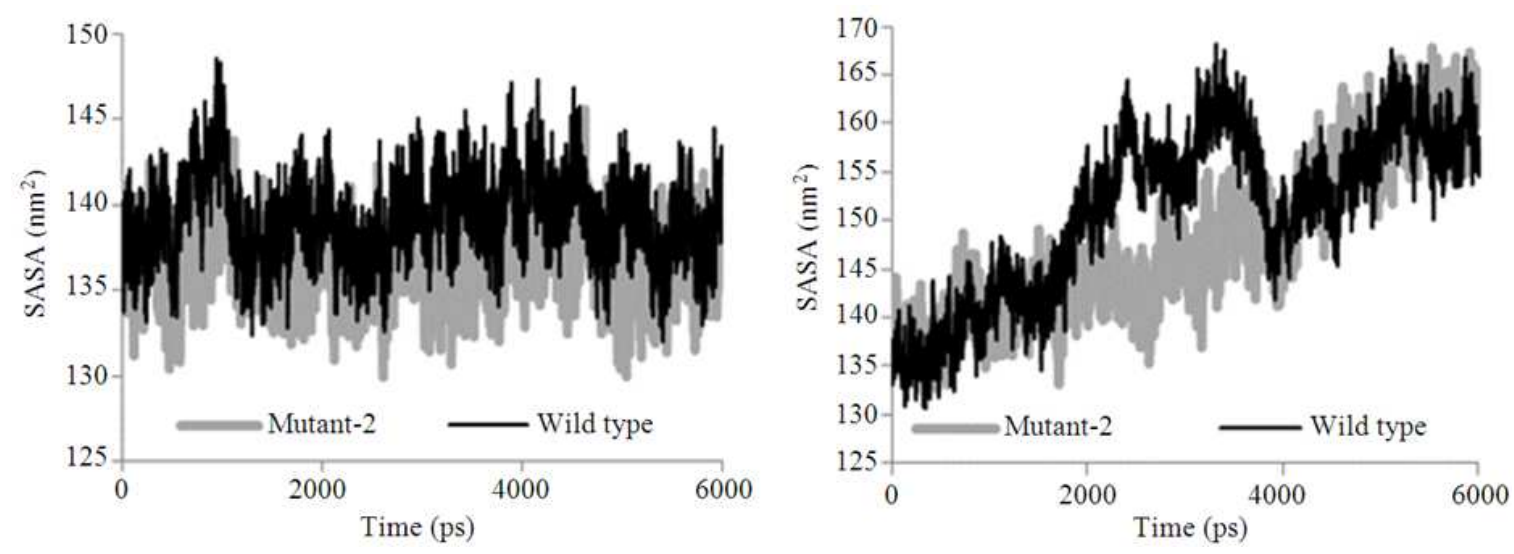

Fig. 8. SASA value of Mutant-2 and wild type CALB $450 \mathrm{~K}$ (up) and $550 \mathrm{~K}$ (down) simulation
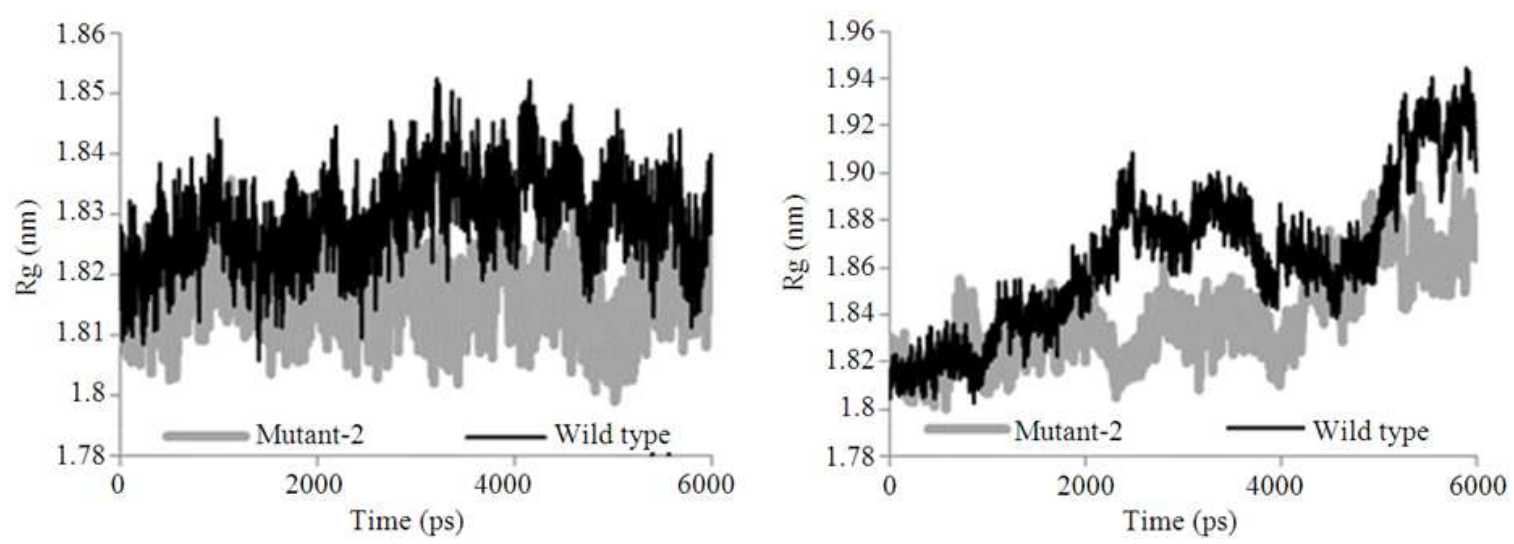

Fig. 9. Rg value of Mutant-2 and wild type CALB $450 \mathrm{~K}$ (up) and $550 \mathrm{~K}$ (down) simulation

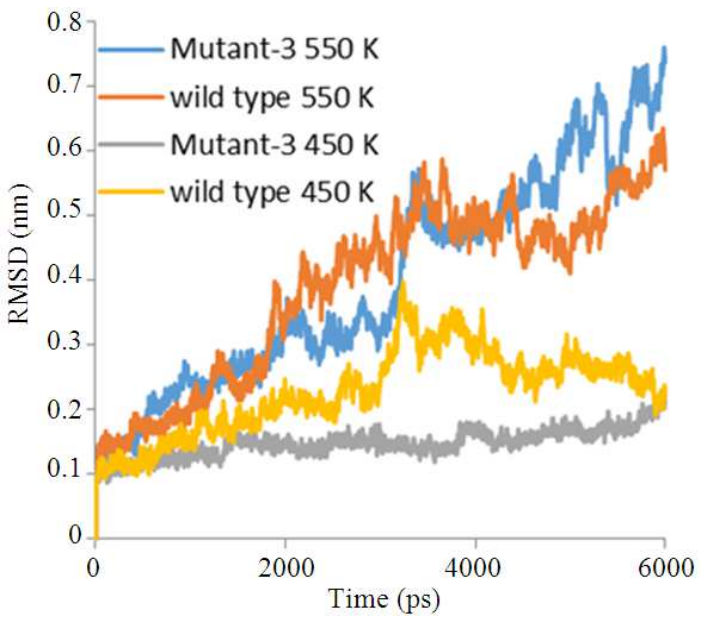

Fig. 10. RMSD value of Mutant-3 and wild type CALB at 450 and $550 \mathrm{~K}$ simulation Mutant-3 at $550 \mathrm{~K}$ (blue), wild type CALB at $550 \mathrm{~K}$ (orange), Mutant-3 at $450 \mathrm{~K}$ (grey), wild type CALB at $450 \mathrm{~K}$ (yellow)

\section{DISCUSSION}

Molecular dynamic simulation is an efficient method that could be utilized for constructing model on protein conformation and time repertoire (Adcock and McCammon, 2006; Ian et al., 2013; Carrillo-Vázquez et al., 2013). As we can see, molecular dynamic simulation is still the most effective way to model the structure and reactivity of an enzyme (Gabel et al., 2009; Lousa et al., 2012; Christensen and Kepp, 2013). In this respect, we have utilized this method since 2011 (Tambunan et al., 2011a; 2011b; 2013; Tambunan and Parikesit, 2012; Parikesit et al., 2013).

Previously, our research group has already produced result on the optimization of enzyme stability, albeit with different types of enzyme (Idrus and Tambunan, 2012). Moreover, we already have experience in conducting mutation study (Tambunan et al., 2012). Hence, we utilized the existing pipeline for this research, albeit with optimization process for CALD. 
Although it is not already wide spread in industrial scale, enzyme stability studies have been conducted extensively in Indonesia (Susilowati et al., 2008; Nurcholis et al., 2012; Hanim et al., 2013). Eventually, the modeling study has been conducted as well (Hertadi et al., 2007). The investment of private companies in Indonesian bio-industry is already available in segmented market (Sukara and SlametLoedin, 2000). To this end, we are optimistic that our developed pipeline will be utilized in local bio-industry. Henceforth, bio-industry needs this pipeline, as the one way to increase productivity is by introducing useful mutation in the enzyme.

The molecular dynamic method has successfully determined the amino acid residues with the higher tendency toward flexibility (Noorbatcha et al., 2010). Thus, this modeling research has successfully sealed down the flexibility tendency, by introducing disulfidebased mutation and increasing the stability of the enzyme. To this end, by using bioinformatics tools, it is possible to design a PCR primer that could introduce the mutation by using site directed mutagenesis method (Wang et al., 2009).

\section{CONCLUSION}

Thermostability analyses of mutants CALB were conducted by using physical parameters such as RMSD, solvent accessible surface area and radius of gyration. These parameters were used to represent structural changes in the process of protein unfolding influenced by temperature. From three mutants generated, the physical parameters showed that all of them might have better thermostability compared to wild type. We proposed the order of mutant thermostability improvement as follows: Mutant-1, Mutant-2 and Mutant-3; with Mutant-1 potentially having better thermostability improvement and Mutant-3, the least stable. This study only predicts the thermostability criteria based on physical properties. Other analysis could be conducted in modeling thermostability improvement; among them is free energy difference between mutant and wild type $(\Delta \Delta \mathrm{G})$.

\section{ACKNOWLEDGEMENT}

This research is supported by Directorate of Research and Community Engagement University of Indonesia (DRPM UI). We thank Dr. Ridla Bakri, Vice Dean of Faculty of Mathematics and Science, University of
Indonesia for his support. Usman Sumo Friend Tambunan contributed to the conception and design of the research experiment and revising the manuscript; Ahmad Randy was involved in designing and carrying out of the experiment, analyzing and interpreting data and helped in drafting the manuscript, while Arli Aditya Parikesit added key materials on the discussion section, rearranged the original manuscript, proofread and improved the manuscript with citation manager.

\section{REFERENCES}

Adcock, S.A. and J.A. McCammon, 2006. Molecular dynamics: Survey of methods for simulating the activity of proteins. Chem. Rev. 106: 1589-615. DOI: $10.1021 / \mathrm{cr} 040426 \mathrm{~m}$

Babakhouya, N., S. Boughrara and F. Abad, 2010. Kinetics and thermodynamics of $\mathrm{Cd}(\mathrm{II})$ ions sorption on mixed sorbents prepared from olive stone and date pit from aqueous solution. Am. J. Environ. Sci., 6: 470-476. DOI: 10.3844/ajessp. 2010.470 .476

Benson, N.C. and V. Daggett, 2008. Dynameomics: Large-scale assessment of native protein flexibility. Protein Sci., 17: 2038-2050.

Betz, S.F., 1993. Disulfide bonds and the stability of globular proteins. Protein Sci., 2: 1551-1558.

Bisht, D., S.K. Yadav and N.S. Darmwal, 2012. Enhanced production of extracellular alkaline lipase by an improved strain of Pseudomonas aeruginosa MTCC 10,055. Am. J. Appl. Sci., 9: 158-167. 10.3844/ajassp.2012.158.167

Bussi, G., D. Donadio and M. Parrinello, 2007. Canonical sampling through velocity rescaling. J. Chem. Phys., 126: 014101. PMID: 17212484

Carrillo-Vázquez, J.P., B. Chimal-Vega, B. ZamoraLópez, L.A. Marchat and C.G. Benítez-Cardoza et al., 2013. Structural consequences of the polymorphism Q223r in the human leptin receptor: A molecular dynamics study. Am. J. Agric. Biol. Sci., 8: 239-248. DOI: 10.3844/ajabssp.2013.239.248

Ceruso, M.A., X. Periole and H. Weinstein, 2004. Molecular dynamics simulations of transducin: Interdomain and front to back communication in activation and nucleotide exchange. J. Mol. Biol., 338: 469-481.

Chandramat, S., N.C. Sekhar and M.G. Adarsh, 2005. A novel video surveillance system based on multimedia messaging service. J. Comput. Sci., 1: 244-248. DOI: 10.3844 jcssp.2005.244.248 
Chodorge, M., L. Fourage, C. Ullmann, V. Duvivier and J.M. Masson et al., 2005. Rational strategies for directed evolution of biocatalysts-application to Candida Antarctica Lipase B (CALB). Adv. Synth. Catal., 347: 1022-1026. DOI: $10.1002 /$ adsc. 200505055

Christensen, N.J. and K.P. Kepp, 2013. Stability mechanisms of a thermophilic laccase probed by molecular dynamics. PLoS One, 8: e61985- e61985. DOI: 10.1371/journal.pone.0061985

Day, R., B.J. Bennion, S. Ham and V. Daggett, 2002. Increasing temperature accelerates protein unfolding without changing the pathway of unfolding. J. Mol. Biol., 322: 189-203. PMID: 12215424

Dombkowski, A.A., 2003. Disulfide by design: A computational method for the rational design of disulfide bonds in proteins. Bioinformatics, 19: 1852-1853. PMID: 14512360

Essman, U., L. Perera, M. Berkowitz, T. Darden and H. Lee et al., 1995. A smooth particle mesh Ewald method. J. Chem. Phys., 103: 8577.

Gabel, F., P. Masson, M.T. Froment, B.P. Doctor and A. Saxena et al., 2009. Direct correlation between molecular dynamics and enzymatic stability: A comparative neutron scattering study of native human butyrylcholinesterase and its "aged" soman conjugate. Biophys. J., 96: 1489-1494.

Ganjalikhany, M.R., B. Ranjbar, A.H. Taghavi and T. Tohidi Moghadam, 2012. Functional motions of Candida Antarctica lipase b: A survey through openclose conformations. PLoS One.

Gundampati, R.K., S. Sahu, K.S. Sonkar, M. Debnath and A.K. Srivastava et al., 2013. Modeling and molecular docking studies on Aspergillus RNAse Niger and Leishmania Donovani actin: Antileishmanial Activity. Am. J. Biochem. Biotechnol., $\quad$ 9: $318-328 . \quad$ DOI: 10.3844/ajbbsp.2013.318.328

Haki, G.D. and S.K. Rakshit, 2003. Developments in industrially important thermostable enzymes: A review. Bioresour. Technol., 89: 17-34. PMID: 12676497

Han, Z., S. Han, S. Zheng and Y. Lin, 2009. Enhancing thermostability of a Rhizomucor miehei lipase by engineering a disulfide bond and displaying on the yeast cell surface. Appl. Microbiol. Biotechnol., 85: 117-126. PMID: 19533118

Hanim, C., L.M. Yusiati, M.N. Cahyanto and A. Wibowo, 2013. Mutagenic improvement of Xylanase production from Xylanolytic bacteria and its phylogenetic analysis. Microbiol. Indones., 7: 51-51.
Harishchander, A., D.A. Anand and S. Senapati, 2010. Analysis of resistance to human immunodeficiency virus protease inhibitors using molecular mechanics and machine learning strategies. Am. Med. J., 1: 126-132. DOI: 10.3844/amjsp.2009.126.132

Hertadi, R., S. Nurbaiti and A. Akhmaloka, 2007. Molecular dynamics analysis of thermostable DNA Pol I ITB-1. Microbiol. Indones., 1: 101-104.

Hess, B., H. Bekker, H.J.C. Berendsen and J.G.E.M. Fraaije, 1997. LINCS: A linear constraint solver for molecular simulations. J. Comput. Chem., 18: 14631472. DOI: 10.1002/(SICI)1096987X(199709)18:12<1463::AID-JCC4>3.0.CO;2-H

Hess, B., C. Kutzner, D. Van Der Spoel and E. Lindahl, 2008. GROMACS 4: Algorithms for highly efficient, load-balanced and scalable molecular simulation. J. Chem. Theory Comput., 4: 435-447. DOI: $10.1021 / \mathrm{ct} 700301 \mathrm{q}$

Humphrey, W., A. Dalke and K. Schulten, 1996. VMD: Visual molecular dynamics. J. Mol. Graph., 14: 33-8.

Ian, I.F., R.T.J. Luis, C.V.J. Pablo, V.S.J. Luis and C.I. Normande et al., 2013. Identification of pharmacological targets combining docking and molecular dynamics simulations. Am. J. Agric. Biol. Sci., 8: 89-106. DOI: 10.3844/ajabssp.2013.89.106

Idrus, S. and U.S.F. Tambunan, 2012. Simulation of riboflavin synthase in Eremothecium gossypii conversion of 6,7-dimethyl-8-ribityllumazine to riboflavin. Online J. Bioinforma., 13: 41-49.

Jeong, M.Y., S. Kim, C.W. Yun, Y.J. Choi and S.G. Cho, 2007. Engineering a de novo internal disulfide bridge to improve the thermal stability of xylanase from Bacillus stearothermophilus No. 236. J. Biotechnol., 127: 300-309. PMID: 16919348

Jorgensen, W.L., J. Chandrasekhar, J.D. Madura, R.W. Impey and M.L. Klein, 1983. Comparison of simple potential functions for simulating liquid water. J. Chem. Phys., 79: 926.

Kim, H.S., Q.A.T. Le and Y.H. Kim, 2010. Development of thermostable lipase B from Candida antarctica (CalB) through in silico design employing B-factor and RosettaDesign. Enzyme Microb. Technol., 47: 1-5.

Kuzmanic, A. and B. Zagrovic, 2010. Determination of ensemble-average pairwise root mean-square deviation from experimental B-factors. Biophys. J., 98: 861-71.

Larsson, D.S.D., L. Liljas and D. Van Der Spoel, 2012. Virus capsid dissolution studied by microsecond molecular dynamics simulations. PLoS Comput. Biol. 
Li, A. and V. Daggett, 1994. Characterization of the transition state of protein unfolding by use of molecular dynamics: Chymotrypsin inhibitor 2. Proc. Nat. Acad. Sci. USA., 91: 10430-10434.

Lim, Y.W., M.H. Ahmad and D.R. Ossen, 2010. Empirical validation of daylight simulation tool with physical model measurement. Am. J. Appl. Sci., 7: 1426-1431. DOI: 10.3844/ajassp.2010.1426.1431

Ling, H.S., T.C. Ling, R. Mohamad and A.B. Ariff, 2009. Characterization of pullulanase type II from Bacillus cereus H1.5. Am. J. Biochem. Biotechnol., 5: 170-179. 10.3844/ajbbsp.2009.170.179

Liu, H.L. and W.C. Wang, 2003. Protein engineering to improve the thermostability of glucoamylase from Aspergillus awamori based on molecular dynamics simulations. Protein Eng., 16: 19-25. PMID: 12646689

Lousa, D., A.M. Baptista and C.M. Soares, 2012. Analyzing the molecular basis of enzyme stability in ethanol/water mixtures using molecular dynamics simulations. J. Chem. Inf. Model, 52: 465-73. DOI: 10.1021/ci200455z

Matsumura, M., G. Signor and B.W. Matthews, 1989. Substantial increase of protein stability by multiple disulphide bonds. Nature, 342: 291-3. PMID: 2812028

Mittelbach, M., 1990. Lipase catalyzed alcoholysis of sunflower oil. J. Am. Oil Chem. Soc., 67: 168-170. DOI: 10.1007/BF02539619

Noorbatcha, I.A., A.M. Khan and H.M. Salleh, 2010. Molecular dynamics studies of human $\beta$ glucuronidase. Am. J. Applied Sci., 7: 823-828. DOI: 10.3844 /ajassp.2010.823.828

Nurcholis, M., N. Nurhayati, I. Helianti, M. Ulfah and B. Wahyuntari et al., 2012. Cloning of $\alpha$ larabinofuranosidase genes and its expression in Escherichia coli: A comparative study of recombinant arabinofuranosidase originatingin Bacillus subtilis DB104 and newly isolated Bacillus licheniformis CW1. Microbiol. Indones., 6: 1-8.

Ong, A.L., A.H. Kamaruddin, S. Bhatia, W.S. Long and S.T. Lim et al., 2006. Performance of free Candida antarctica lipase $\mathrm{B}$ in the enantioselective esterification of (R)-ketoprofen. Enzyme Microb. Technol., 39: 924-929.

Parikesit, A.A., Kinanty and U.S.F. Tambunan, 2013. Screening of commercial cyclic peptides as inhibitor envelope protein Dengue Virus (DENV) through molecular docking and molecular dynamics. Pak. J. Biol. Sci., 16: 1836-1848.
Paschek, D., R. Day and A.E. García, 2011. Influence of water-protein hydrogen bonding on the stability of Trp-cage miniprotein. A comparison between the TIP3P and TIP4P-Ew water models. Phys. Chem. Chem. Phys., 13: 19840-7. PMID: 21845272

Patel, R.N., A. Banerjee, V. Nanduri, A. Goswami and F.T. Comezoglu, 2000. Enzymatic resolution of racemic secondary alcohols by lipase B from Candida antarctica. J. Am. Oil Chem. Soc., 77: 1015-1019. DOI: 10.1007/s11746-000-0161-y

Payne, C.M., M.G. Resch, L. Chen, M.F. Crowley and M.E. Himmel et al., 2013. Glycosylated linkers in multimodular lignocellulose-degrading enzymes dynamically bind to cellulose. Proc. Nat. Acad. Sci. USA., 110: 14646-51. PMID: 23959893

Peeters, J.W., O. Van Leeuwen, A.R.A. Palmans and E.W. Meijer, 2005. Lipase-catalyzed ring-opening polymerizations of 4-substituted $\varepsilon$-caprolactones: Mechanistic considerations. Macromolecules, 38: 5587-5592. DOI: 10.1021/ma050510j

Pikkemaat, M.G., A.B.M. Linssen, H.J.C. Berendsen and D.B. Janssen, 2002. Molecular dynamics simulations as a tool for improving protein stability. Protein Eng., 15: 185-92. PMID: 11932489

Purmonen, M., J. Valjakka, K. Takkinen, T. Laitinen and J. Rouvinen, 2007. Molecular dynamics studies on the thermostability of family 11 xylanases. Protein Eng. Des. Sel., 20: 551-9. PMID: 17977846

Sandström, A.G., K. Engström, J. Nyhlén, A. Kasrayan and J.E. Bäckvall, 2009. Directed evolution of Candida antarctica lipase A using an episomaly replicating yeast plasmid. Protein Eng. Des. Sel., 22: 413-20. PMID: 19509064

Santarossa, G., P.G. Lafranconi, C. Alquati, L. DeGioia and L. Alberghina et al., 2005. Mutations in the "lid" region affect chain length specificity and thermostability of a Pseudomonas fragi lipase. FEBS Lett., 579: 2383-6. PMID: 15848176

Senn, H.M. and W. Thiel, 2009. QM/MM methods for biomolecular systems. Angew. Chem. Int. Ed. Eng., 48: 1198-229. PMID: 19173328

Sharma, R., Y. Chisti and U.C. Banerjee, 2001. Production, purification, characterization and applications of lipases. Biotechnol. Adv., 19: 62762. PMID: 14550014

Van Der Spoel, D., E. Lindahl, B. Hess, G. Groenhof and A.E. Mark et al., 2005. GROMACS: Fast, flexible and free. J. Comput. Chem., 26: 1701-18. PMID: 16211538 
Subramanian, S. and R. Natarajan, 2011. A tool for generation and minimization of test suite by mutant gene algorithm. J. Comput. Sci. 7: 1581-1589. DOI: 10.3844/jcssp.2011.1581.1589

Suen, W.C., N. Zhang, L. Xiao, V. Madison and A. Zaks, 2004. Improved activity and thermostability of Candida antarctica lipase B by DNA family shuffling. Protein Eng. Des. Sel., 17: 133-40. PMID: 15047909

Sukara, E. and I. Slamet-Loedin, 2000. Agricultural Biotechnology in Indonesia. p. 1. In Biotechnology Research and Policy: Needs and Priorities in the Context of Southeast Asia's Agricultural Objectives.

Susilowati, P.E., P. Aditiawati, F. Madayanti and A. Akhmaloka, 2008. The effect of mutation at Thr 295 of Saccharomyces cerevisiae eRF1 on suppression of nonsense Codons and eRF1 structure. Microbiol. Indones., 2: 11-16.

Tambunan, U.S.F., N. Apriyanti, A.A. Parikesit, W. Chua and K. Wuryani, 2011a. Computational design of disulfide cyclic peptide as potential inhibitor of complex NS2B-NS3 dengue virus protease. African J. Biotechnol., 10: 12281-12290.

Tambunan, U.S.F., D. Febrianto and A.A. Parikesit, 2012. In silico genetic variation pathogenicity analysis of hemagglutinin, matrix 1 and non structural 1 protein of human $\mathrm{H} 5 \mathrm{~N} 1$ Indonesian Strain. IIOABJ, 3: 5-14.

Tambunan, U.S.F., R.S. Noors and A.A. Parikesit, 2011b. Molecular dynamics simulation of DENV RNA-dependent RNA-polymerase with potential inhibitor of disulfide cyclic peptide. Online J. Biol. Sci., 11: 48-62. DOI: 10.3844/ojbsci.2011.48.62

Tambunan, U.S.F. and A.A. Parikesit, 2012. HPV Bioinformatics: In Silico Detection, Drug Design and Prevention Agent Development. In: Topics on Cervical Cancer with an Advocacy for Prevention, Rajkumar, R. (Ed.)., Intech Publishing, Rijeka, Croatia, p: 237-252.

Tambunan, U.S.F., M.R. Rahdiansyah and A.A. Parikesit, 2013. In silico design of the M2 proton channel inhibitors of H1N1 virus. Online J. Biol. Sci., 13: 1-12. DOI: 10.3844/ojbsci.2013.1.12

Uppenberg, J., M.T. Hansen, S. Patkar and T.A. Jones, 1994. The sequence, crystal structure determination and refinement of two crystal forms of lipase B from Candida antarctica. Structure, 2: 293-308. PMID: 8087556
Vijayan, R., N. Subbarao and B.N. Mallick, 2007. In silico modeling of $\alpha 1 \mathrm{~A}$-adrenoceptor: Interaction of its normal and mutated active sites with noradrenaline as well as its agonist and antagonist. Am. J. Biochem. Biotechnol., 3: 216224. DOI: 10.3844/ajbbsp.2007.216.224

Wakarchuk, W.W., W.L. Sung, R.L. Campbell, A. Cunningham and D.C. Watson et al., 1994. Thermostabilization of the Bacillus circulans xylanase by the introduction of disulfide bonds. Protein Eng., 7: 1379-86. PMID: 7700870

Wang, J., P. Cieplak and P.A. Kollman, 2000. How well does a Restrained Electrostatic Potential (RESP) model perform in calculating conformational energies of organic and biological molecules? J. Comput. Chem., 21: 1049-1074. DOI: $\quad 10.1002 / 1096-$ 987X(200009)21:12<1049::AIDJCC3>3.0.CO;2$\mathrm{F}$

Wang, J., R.M. Pielak, M.A. McClintock and J.J. Chou, 2009. Solution structure and functional analysis of the influenza B proton channel. Nat. Struct. Mol. Biol., 16: 1267-71.

Xu, Y., W. Du, D. Liu and J. Zeng, 2003. A novel enzymatic route for biodiesel production from renewable oils in a solvent-free medium. Biotechnol. Lett., 25: 1239-41. PMID: 14514074

Yamaguchi, S., K. Takeuchi, T. Mase, K. Oikawa and T. McMullen et al., 1996. The consequences of engineering an extra disulfide bond in the Penicillium camembertii mono- and diglyceride specific lipase. Protein Eng., 9: 789-95. PMID: 8888145

Yan, C., V. Pattani, J.W. Tunnell and P. Ren, 2010. Temperature-induced unfolding of Epidermal Growth Factor (EGF): Insight from molecular dynamics simulation. J. Mol. Graph. Model., 29: 2- 12 .

Zhao, X., B. El-Zahab, R. Brosnahan, J. Perry and P. Wang, 2007. An organic soluble lipase for waterfree synthesis of biodiesel. Appl. Biochem. Biotechnol., 143: 236-43. PMID: 18057451

Zhao, L., J. Wang, X. Dou and Z. Cao, 2009. Studying the unfolding process of protein $\mathrm{G}$ and protein $\mathrm{L}$ under physical property space. BMC Bioinformat., 10: S44. 